\section{Role of the processing pore of the ClpX AAA+ ATPase in the recognition and engagement of specific protein substrates}

\author{
Samia M. Siddiqui, ${ }^{1}$ Robert T. Sauer, ${ }^{1}$ \\ and Tania A. Baker ${ }^{1,2,3}$ \\ ${ }^{1}$ Massachusetts Institute of Technology, Department
of Biology, ${ }^{2}$ Howard Hughes Medical Institute,
Cambridge, Massachusetts 02139, USA
}

ClpX binds substrates bearing specific classes of peptide signals, denatures these proteins, and translocates them through a central pore into $\mathrm{ClpP}$ for degradation. $\mathrm{ClpX}$ with the V154F pore mutation is severely defective in binding substrates bearing C-motif 1 degradation signals and is also impaired in a subsequent step of substrate engagement. In contrast, this mutant efficiently processes substrates with other classes of recognition signals both in vitro and in vivo. These results demonstrate that the $\mathrm{ClpX}$ pore functions in the recognition and catalytic engagement of specific substrates, and that $\mathrm{ClpX}$ recognizes different substrate classes in at least two distinct fashions.

Received November 17, 2003; revised version accepted December 30, 2003.

Energy-dependent protein degradation plays an important role in many cellular processes. In Escherichia coli, there are five proteases - ClpXP, ClpAP, HslUV, FtsH, and Lon - that carry out ATP-dependent intracellular proteolysis (Hengge and Bukau 2003). Each of these proteases contains a AAA or AAA+ ATPase and a compartmentalized peptidase. In ClpXP, for example, ClpX is a hexameric-ring ATPase, and ClpP is a barrel-shaped peptidase formed by the back-to-back stacking of two heptameric rings (Wawrzynow et al. 1995; Wang et al. 1997). The active sites of ClpP are located within the barrel, accessible only by passage through a narrow axial portal too small to admit native proteins. For ClpXP to function in protein degradation, ClpX must initially recognize native substrates, then denature these molecules, and finally translocate the unfolded polypeptide into ClpP for degradation (Kim et al. 2000; Singh et al. 2000; Fig. 1A). The specificity of degradation by ClpXP is determined at the initial step of substrate recognition by ClpX. ClpX can also function by itself as a disassembly chaperone, using its protein-unfoldase activity to dis-

[Keywords: AAA ATPase; peptide binding; protein unfolding; protein translocation]

${ }^{3}$ Corresponding author.

E-MAIL tabaker@mit.edu; FAX (617) 252-1852.

Article and publication are at http://www.genesdev.org/cgi/doi/10.1101/ gad.1170304 mantle macromolecular complexes (Levchenko et al. 1995; Kruklitis et al. 1996).

ClpX recognizes substrates by binding to peptide signals that are generally exposed at either the amino- or carboxy-terminal end of an otherwise native protein. Proteomic studies of ClpXP substrates in Escherichia coli led to the discovery of five distinct classes of peptide signals that mediate ClpXP degradation of attached proteins (Flynn et al. 2003). Two of these recognition signals, or "tags", are found at the carboxyl terminus of substrates (C-motif 1 and C-motif 2), whereas the remaining three are amino-terminal (N-motif 1, N-motif 2, and $\mathrm{N}$-motif 3). The ssrA tag, a C-motif 1 signal, is one of the most efficient and best-characterized recognition signals. This 11-residue peptide is added cotranslationally to nascent polypeptides when ribosomes stall (Keiler et al. 1996). Attachment of this signal to any protein renders it a substrate for degradation by ClpXP /Gottesman et al. 1998). SsrA-tagged substrates are also recognized by $\mathrm{SspB}$, an adaptor/delivery protein that enhances their binding to and degradation by ClpXP (Levchenko et al. 2000). Although the kinds of recognition signals that interact with ClpX are reasonably well characterized, major questions remain about how such signals are recognized. Do all classes of recognition motifs interact with a common site, or are there distinct sites for different substrates? Where in the hexameric ClpX enzyme do these sites reside?

A prominent feature of the $\mathrm{Clp} / \mathrm{Hsp} 100$ ATPases is a central pore coincident with the sixfold axis of the hexamer (Fig. 1B). Electron microscopy of ClpXP shows that this pore in ClpX aligns with the axial portal of ClpP, forming a narrow, but continuous channel that provides access to the degradation chamber of $\mathrm{ClpP}$ (Ortega et al. 2000). Hence, the ClpX pore must play a role in the translocation of denatured substrates into ClpP. Furthermore, as ssrA-tagged substrates are translocated/ degraded by ClpXP (and the related ClpAP protease) in a directional fashion starting with the degradation tag (Weber-Ban et al. 1999; Lee et al. 2001), the ssrA tag likely enters the ClpX protein-processing pore at an early stage in the reaction. These observations raise the possibility that the pore may function in recognition of substrates as well as in the subsequent unfolding and translocation steps. In a model of the ClpX hexamer based on the crystal structure of Helicobacter pylori ClpX (Kim and Kim 2003), the central pore contains a GYVG sequence motif, which is highly conserved in other ClpX enzymes and in related Clp/Hsp100 ATPases (Wang et al 2001; Fig. 1B,C). Interestingly, in different structures of $\mathrm{HslU}$ (which is $\sim 50 \%$ identical in sequence to $\mathrm{ClpX}$ ), the size of the pore and the conformation of the GYVG motif vary as a function of nucleotide state (Wang et al. 2001), suggesting a dynamic role for these residues in the protease reaction cycle of ClpXP as well. Mutagenesis studies in HslU suggest that the bulky hydrophobic residues within this motif may interact with the substrate protein during translocation (Wang et al. 2001).

In the work reported here, we have tested mutations in the E. coli ClpX pore for effects on enzyme activity in vivo and in vitro. Several ClpX variants with substitutions for the valine in the GYVG motif displayed differential abilities to process substrates in vivo, whereas 


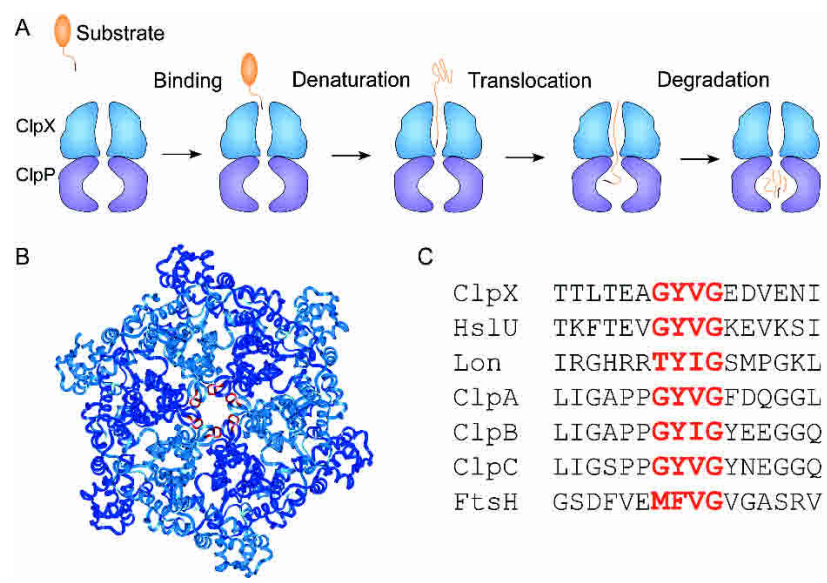

Figure 1. (A) Substrates for ClpXP degradation are bound by ClpX via peptide recognition tags, unfolded, and translocated into ClpP for degradation. $(B)$ Position of the conserved pore motif GYVG (highlighted in red) in the ClpX hexamer. $(C)$ Conservation of the pore motif in ClpX and related ATPases.

only a conservative substitution for the tyrosine supported activity. Substitution of the valine with phenyl-

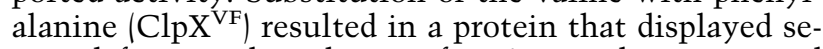
vere defects in degradation of ssrA-tagged proteins and other C-motif 1 substrates in vitro, but only modest changes in degradation of other classes of substrates. The defect in degradation of ssrA-tagged proteins arises predominantly from a reduced ability of $\mathrm{ClpX}^{\mathrm{VF}}$ to bind these substrates, but also results from inefficient engagement of bound substrate. These results reveal that the protein-processing pore of ClpX plays a role in the recognition and engagement of C-motif 1 substrates, and indicate that there is more than one site of interaction on ClpX for the different classes of substrates.

\section{Results and Discussion}

\section{ClpX pore mutants have differential activity in vivo}

As an initial step in understanding the importance of the GYVG motif in the ClpX pore, we engineered mutants with a range of amino acid substitutions at Y153 and V154 and screened these mutants using two biological assays for $\mathrm{ClpX}$ function, phage $\mathrm{Mu}$ growth and restriction alleviation. For bacteriophage Mu to grow in E. coli, ClpX must disassemble a hyperstable MuA transposaseDNA complex, an activity that requires the unfolding of at least one MuA subunit (Burton and Baker 2003). MuA carries a C-motif $2 \mathrm{ClpX}$ recognition signal (Flynn et al. 2003). Restriction alleviation in E. coli, a process that transiently suppresses DNA restriction following damage, requires ClpXP degradation of the HsdR subunit of the EcoK restriction enzyme (Makovets et al. 1999). HsdR has an amino-terminal ClpX-binding sequence (Flynn et al. 2003), but how this protein is recognized for degradation is not well understood.

The biological assays for ClpX function (Fig. 2) revealed a range of activities for the Y153 and V154 substitutions and suggested a substrate-specific role in protein recognition or processing for some mutants. All of the V154 substitutions tested (alanine, cysteine, leucine, phenylalanine, tryptophan, asparagine, aspartic acid, and lysine) supported $\mathrm{Mu}$ growth, but only four of these enzymes (V154A, V154C, V154W, and V154K) were active in restriction alleviation (Fig. 2A). Of the Y153 substitutions tested, only the tryptophan mutant was biologically active (Fig. 2B). Taken together, these results show that the identity of the 153 and 154 side chains in ClpX are important in determining its intracellular activity, and reveal that some mutant variants are fully active in one assay but inactive in the other. Because the two assays probe the interaction of $\mathrm{ClpX}$ with distinct substrates, one explanation for these results is that the ClpX pore plays a role in the differential recognition of protein substrates. The experiments discussed below test this hypothesis.

\section{Properties of purified pore mutants}

We purified two of the ClpX pore mutants (V154F and Y153A) for biochemical investigations of the role of the pore in ClpX in substrate recognition and protein processing. The basal rate of ATP hydrolysis for the V154F mutant $\left(\mathrm{ClpX}^{\mathrm{VF}}\right)$ was within error of the value for the wild-type enzyme $\left(225 \pm 6 \mathrm{~min}^{-1}\right.$ compared with $207 \pm 17 \mathrm{~min}^{-1}$ ), and the ATPase activity of the Y153A mutant $\left(\mathrm{ClpX}^{\mathrm{YA}}\right)$ was roughly two-thirds that of wildtype ClpX $\left(138 \pm 7 \mathrm{~min}^{-1}\right)$. Clearly, both mutant proteins retain the ability to hydrolyze ATP at near wild-type levels, suggesting that the structures of the enzymes were not affected in any gross fashion by the mutations. Both mutant proteins also assembled into hexamers and interacted with $\mathrm{ClpP}$ (data not shown).
A
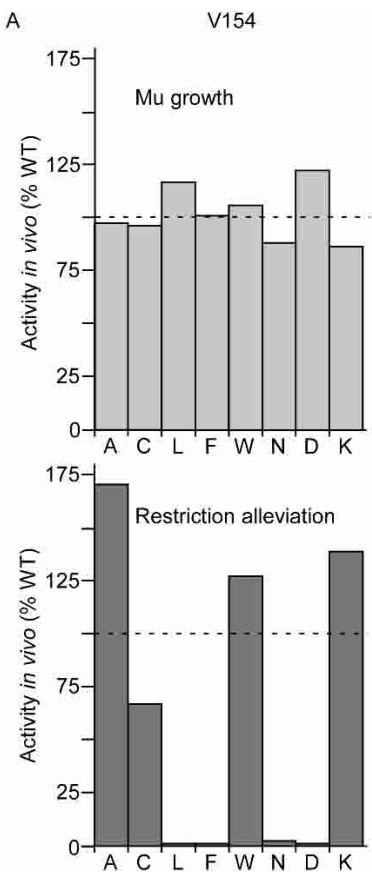

B
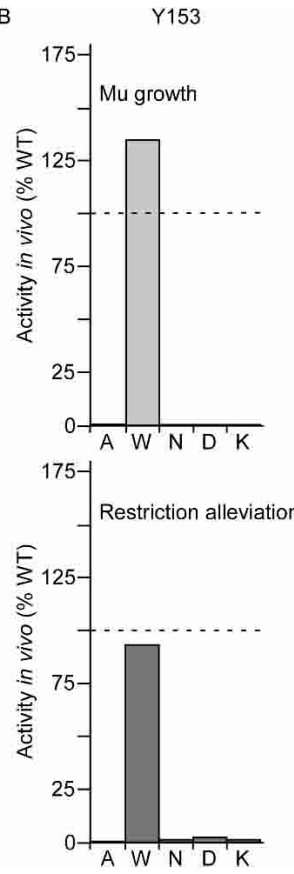

Figure 2. Biological activity of $\mathrm{ClpX}$ mutants with substitutions for $\operatorname{Val}^{154}(A)$ or $\operatorname{Tyr}^{153}(B)$ in supporting phage Mu growth and restriction alleviation. For $\mathrm{Mu}$ growth, activity was defined as the number of phage plaques obtained for each mutant strain, normalized by the number of plaques obtained from cells containing wildtype ClpX. For restriction alleviation, activity was determined by assaying the plating efficiency of unmodified $\lambda$ phage on a restriction-competent strain treated with 2-aminopurine as described (Kim et al. 2001). 
$C l p X^{V F}$ pore mutant shows substrate-specific defects in vitro

As discussed above, the $\mathrm{ClpX}^{\mathrm{VF}}$ mutant displayed a differential ability to process MuA and HsdR substrates in vivo. To investigate the ability of this mutant to process substrates with different types of recognition sequences in vitro, the purified $\mathrm{ClpX}^{\mathrm{VF}}$ protein was combined with $\mathrm{ClpP}$ and tested for its ability to degrade hybrid proteins containing Arc repressor fused to one of five different classes of ClpX substrate-recognition signals. The fusion proteins contained $\mathrm{ClpX}$ recognition signals at either the carboxyl terminus (C-motif 1: Arc-ssrA; or C-motif 2: Arc-MuA) or amino terminus (N-motif 1: $\lambda \mathrm{O}$-Arc; $\mathrm{N}$ motif 2: IscS-Arc; or N-motif 3: DksA-Arc). Previous studies have established that each of these Arc fusion proteins is specifically degraded by ClpXP, whereas the parental Arc protein is not a ClpXP substrate (Flynn et al. 2003).

When combined with $\mathrm{ClpP}$, the $\mathrm{ClpX} \mathrm{V}^{\mathrm{VF}}$ mutant was severely defective in degrading Arc-ssrA (Fig. 3A), dis-

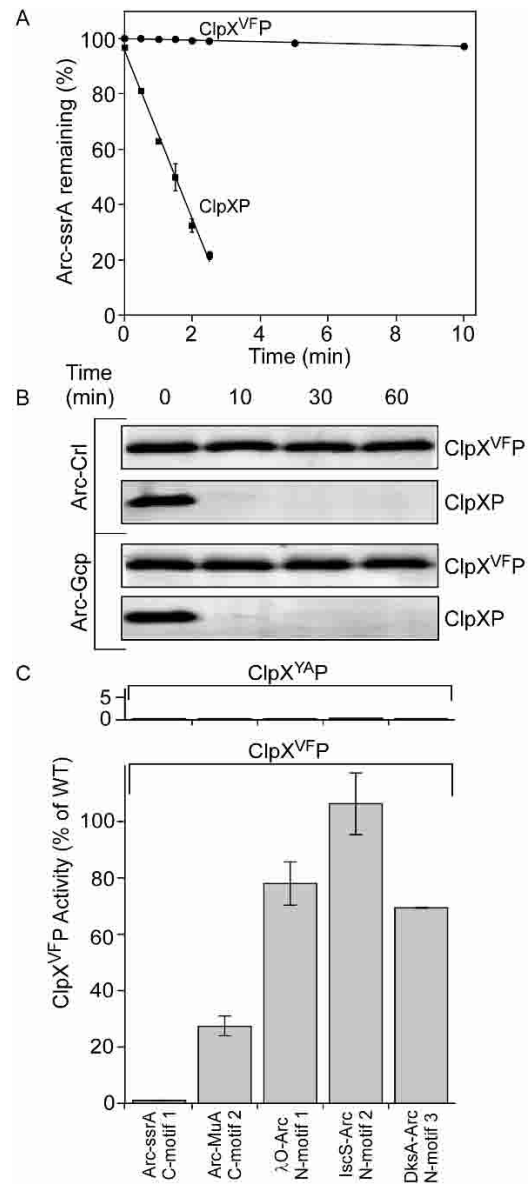

Figure 3. Degradation of purified substrate proteins. $(A)$ Degradation of the C-motif 1 substrate $\left[{ }^{35} \mathrm{~S}\right]$ Arc-ssrA $(5 \mu \mathrm{M})$ by $\mathrm{ClpX}{ }^{\mathrm{VF}} \mathrm{P}$ or wild-type ClpXP monitored by release of acid-soluble radioactivity. (B) Degradation of the C-motif 1 substrates, Arc-Crl and Arc-Gcp, by $\mathrm{ClpX}^{\mathrm{VF} P}$ and wild-type ClpXP monitored by SDS-PAGE. $(C)$ Normalized degradation rates (determined from the initial linear phase of the degradation curve) of Arc fusion proteins $(5 \mu \mathrm{M})$ with five classes of degradation signals by $\mathrm{ClpX}^{\mathrm{VF}} \mathrm{P}$ relative to wild-type ClpXP. For IscS-Arc and DksA-Arc, similar results were also obtained when the substrate concentration was increased to $20 \mu \mathrm{M}$.

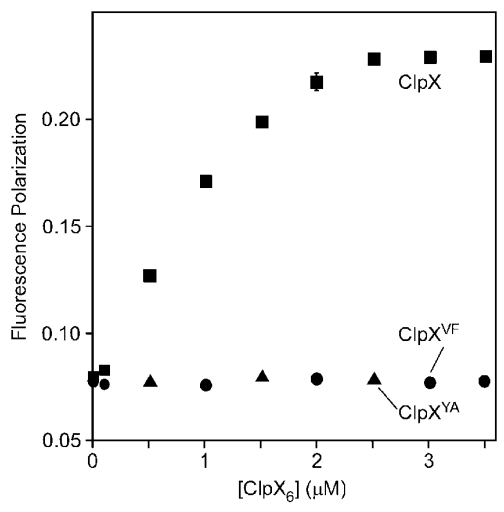

Figure 4. Wild-type ClpX binds to a peptide (100 nM) containing an ssrA degradation tag as assayed by fluorescence polarization, but the V154F and Y153A pore mutants of ClpX show no significant binding.

playing only a small fraction of wild-type activity. $\mathrm{ClpX}^{\mathrm{VF}} \mathrm{P}$ was also defective in degrading other C-motif 1 substrates including Arc-Crl (Fig. 3B), Arc-Gcp (Fig. 3B), and GFP-ssrA (data not shown). However, $\mathrm{ClpX}^{\mathrm{VF}} \mathrm{P}$ degraded the four remaining classes of Arc fusion substrates with relative activities of roughly $30 \%$ for the C-motif 2 substrate and $70 \%-100 \%$ for substrates bearing one of the three $\mathrm{N}$-motif recognition signals (Fig. 3C). In these assays, the substrate concentrations were below saturating levels, and thus, activity is a function of both $\mathrm{K}_{\mathrm{m}}$ and $\mathrm{V}_{\max }$ (see legend to Fig. 3C). Hence, these results demonstrate that the ClpX ${ }^{\mathrm{VF}}$ mutant is able to bind and catalyze the denaturation and translocation of substrates with most classes of degradation signals with reasonable efficiencies. In contrast, the extremely low activity of the $\mathrm{ClpX}^{\mathrm{VF}}$ mutant in degrading substrates with C-motif 1 recognition signals is likely to result from a specific defect in the initial binding or subsequent processing of these substrates (see below).

To test directly for binding of the $\mathrm{ClpX}^{\mathrm{VF}}$ variant to the ssrA-degradation tag, we used a fluorescently labeled ssrA peptide and assayed binding by changes in fluorescence polarization (Fig. 4). In these experiments, wildtype ClpX bound the ssrA-tag peptide half-maximally at a concentration near $1 \mu \mathrm{M}$, a value expected from previous studies (Wah et al. 2002), but $\mathrm{ClpX}^{\mathrm{VF}}$ showed no significant binding at the concentrations tested. On the basis of this experiment and the degradation results, we conclude that the V154F mutation specifically impairs recognition and initial binding of ssrA-tagged proteins and other C-motif 1 substrates to ClpX.

The VF pore mutation increases $K_{m}$ for degradation of Arc-ssrA

Although feeble, a small amount of $\mathrm{ClpX} \mathrm{VF}^{\mathrm{VF}} \mathrm{P}$ degradation of Arc-ssrA was observed (Fig. 3A). This low level of activity allowed us to measure degradation rates over a range of substrate concentrations to determine whether the degradation defect could be suppressed by high concentrations of substrate. As shown in Figure 5A, ArcssrA was degraded more rapidly by $\mathrm{ClpX}^{\mathrm{VF}} \mathrm{P}$ at higher substrate concentrations. These kinetic data fit well to a Michaelis-Menten model with a $\mathrm{K}_{\mathrm{m}}$ of $110 \pm 20 \mu \mathrm{M}$ and a $\mathrm{V}_{\max }$ of $1.8 \pm 0.3 \mathrm{~min}^{-1} / \mathrm{ClpX}_{6}$. By comparison, degra- 

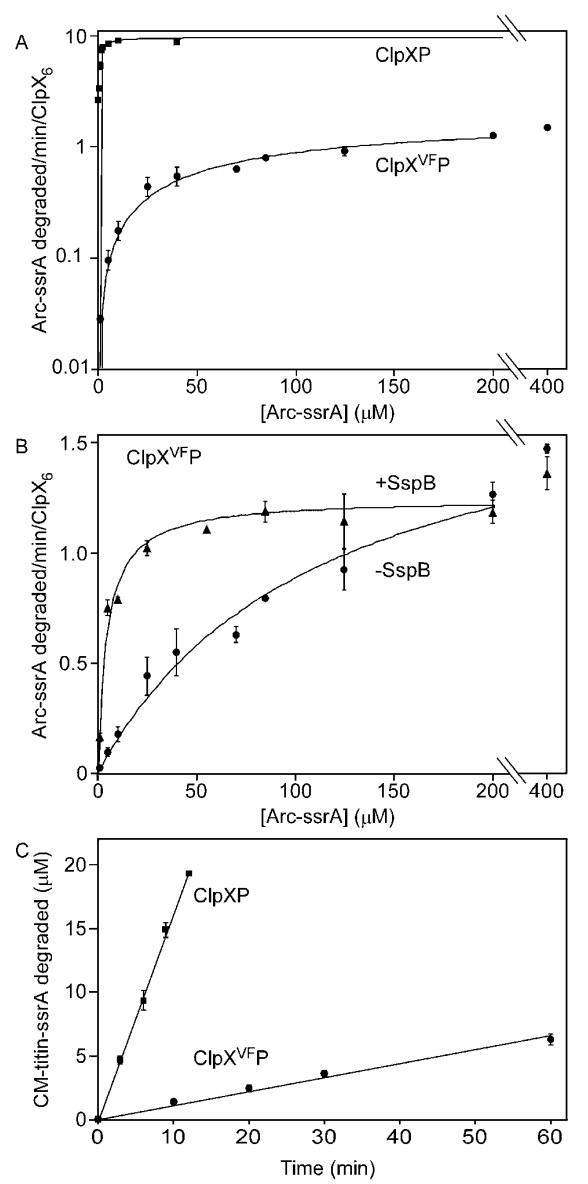

Figure 5. Kinetics of degradation of ssrA-tagged substrates. $(A)$ Degradation of different concentrations of $\left[{ }^{35} \mathrm{~S}\right]$ Arc-ssrA by wildtype ClpXP $\left(0.1 \mu \mathrm{M} \mathrm{ClpX}_{6} ; 0.3 \mu \mathrm{M} \mathrm{ClpP}_{14}\right)$ or $\mathrm{ClpX}^{\mathrm{VF}} \mathrm{P}(0.3 \mu \mathrm{M}$ $\mathrm{ClpX}_{6}{ }^{\mathrm{VF}} ; 0.8 \mu \mathrm{M} \mathrm{ClpP}_{14}$ ) assayed by release of acid-soluble radioactive peptides. Fits to the Michaelis-Menten equation gave kinetic parameters for the wild-type $\left(\mathrm{K}_{\mathrm{m}}=0.70 \pm 0.15 \mu \mathrm{M}_{;} \mathrm{V}_{\max }=9.7 \pm 0.5\right.$ $\left.\mathrm{min}^{-1} / \mathrm{ClpX}_{6}\right)$ and mutant enzymes $\left(\mathrm{K}_{\mathrm{m}}=110 \pm 20 \mu \mathrm{M}_{i}\right.$ $\left.\mathrm{V}_{\max }=1.8 \pm 0.3 \min ^{-1} / \operatorname{ClpX}_{6}{ }^{\mathrm{VF}}\right)$. The $\mathrm{V}_{\max }$ value for wild-type ClpXP is higher than reported previously (Burton et al. 2001), because a lower salt-degradation buffer was used here. $(B)$ Degradation of Arc-ssrA by ClpX $\mathrm{X}^{\mathrm{VF}} \mathrm{P}$ (same conditions as in $A$ ) with or without $0.5 \mu \mathrm{M}$ of $\mathrm{SspB}$. With $\mathrm{SspB}, \mathrm{K}_{\mathrm{m}}$ was $5 \pm 1 \mu \mathrm{M}$ and $\mathrm{V}_{\max }$ was $1.3 \pm 0.3$ $\mathrm{min}^{-1} / \mathrm{ClpX}_{6}{ }^{\mathrm{VF}} .(C)$ Degradation of the denatured substrate $\left[{ }^{35} \mathrm{~S}\right] \mathrm{CM}$ titin-ssrA $(50 \mu \mathrm{M})$ by $\mathrm{ClpXP}\left(0.3 \mu \mathrm{M} \mathrm{ClpX}_{6} ; 0.8 \mu \mathrm{M} \mathrm{ClpP}_{14}\right)$ or $\mathrm{ClpX}^{\mathrm{VF}} \mathrm{P}\left(0.3 \mu \mathrm{M} \mathrm{ClpX}_{6}{ }^{\mathrm{VF}} ; 0.8 \mu \mathrm{M} \mathrm{ClpP}_{14}\right)$ in the presence of SspB $(0.5 \mu \mathrm{M})$.

dation of Arc-ssrA mediated by wild-type ClpXP under the same conditions showed $\mathrm{K}_{\mathrm{m}}$ of $0.70 \pm 0.15 \mu \mathrm{M}$ and $\mathrm{V}_{\max }$ of $9.7 \pm 0.5 \mathrm{~min}^{-1} / \mathrm{ClpX}_{6}\left(\mathrm{~V}_{\max }\right.$ for ClpXP degradation of Arc-ssrA is higher in the degradation buffer used here than reported previously; Burton et al. 2001). Hence, the defect observed for $\mathrm{ClpX}^{\mathrm{VF}}$-mediated degradation of Arc-ssrA results from a 150 -fold increase in $\mathrm{K}_{\mathrm{m}}$ and a 5.4-fold decrease in $\mathrm{V}_{\max }$. Because $\mathrm{K}_{\mathrm{m}}$ for ClpXP degradation of ssrA-tagged substrates is essentially equal to $\mathrm{K}_{\mathrm{d}}$ for binding (Kenniston et al. 2003), these experiments provide additional evidence that the pore mutation in $\mathrm{ClpX}^{\mathrm{VF}}$ causes a specific defect in the binding of C-motif 1 substrates.

The substrate delivery protein SspB enhances degradation of ssrA-tagged substrates by wild-type ClpXP, principally by strengthening binding and lowering $\mathrm{K}_{\mathrm{m}}$
(Levchenko et al. 2000). In the presence of SspB, the $\mathrm{K}_{\mathrm{m}}$ for degradation of Arc-ssrA by ClpX ${ }^{\mathrm{VF}} \mathrm{P}$ was decreased to $5 \pm 1 \mu \mathrm{M}$ (Fig. $5 \mathrm{~B}$ ) with a $\mathrm{V}_{\max }$ value of $1.2 \pm 0.3 \mathrm{~min}^{-1} /$ ClpX ${ }_{6}$. Although SspB lowered $\mathrm{K}_{\mathrm{m}}$ for Arc-ssrA degradation more than 20-fold, the observed value was still roughly 10 - to 20-fold higher than the corresponding value for wild-type ClpXP in the presence of SspB (Levchenko et al. 2000). These results further support the conclusion that the $\mathrm{ClpX}^{\mathrm{VF}}$ mutant binds poorly to ssrAtagged substrates, as binding remains significantly worse than wild type, even when assisted by a specialized delivery protein.

\section{ClpX $X^{V F}$ is also defective in processing C-motif 1 substrates}

The experiments described above show that the maximal velocity of $\mathrm{ClpX}{ }^{\mathrm{VF}} \mathrm{P}$ degradation of Arc-ssrA is significantly lower than for wild-type ClpXP. This defect could arise, for example, because ssrA-tagged substrates are denatured or translocated more slowly by the V154F mutant. To test whether protein unfolding was the major step affected, we assayed degradation of a chemically modified substrate protein (CM-titin-ssrA) that is denatured in solution, and thus, does not require enzymatic unfolding (Kenniston et al. 2003). Under conditions of substrate saturation (50 $\mu \mathrm{M}$ CM-titin-ssrA; $0.5 \mu \mathrm{M}$ $\mathrm{SspB}$ ), degradation of CM-titin-ssrA by $\mathrm{ClpX}^{\mathrm{VF}} \mathrm{P}$ still occurred significantly more slowly than degradation by wild-type ClpXP (Fig. 5C). Thus, the slow degradation observed with the mutant enzyme is not suppressed by using a denatured substrate. We conclude that the $\mathrm{V}_{\max }$ defect of the $\mathrm{ClpX}^{\mathrm{VF}}$ variant affects an enzymatic step that is required for degradation of both native and denatured C-motif 1 substrates (see below).

\section{Degradation and substrate-binding defects of the ClpX ${ }^{Y A}$ pore mutant}

In combination with $\mathrm{ClpP}, \mathrm{ClpX}$ bearing the Y153A pore mutation was unable to degrade Arc fusion substrates with any of the five recognition motifs (Fig. 3C, top). Moreover, we detected no binding of the $\mathrm{ClpX}^{\mathrm{YA}}$ mutant to the ssrA peptide in the fluorescence polarization assay (Fig. 4). Because this mutant was also inactive both in MuA disassembly and HsdR degradation in vivo, it appears to be defective in the binding, denaturation, and/or translocation of substrates with many different classes of ClpX recognition signals. This type of global defect is often indicative of aberrant folding. However, as noted above, the $\mathrm{ClpX}^{\mathrm{YA}}$ mutant displayed substantial ATPase activity, formed hexamers, and bound ClpP in gel-filtration experiments (data not shown). As a result, if the global degradation defects of the Y153A mutant are caused by structural disruptions, then these perturbations must not be propagated to the sites required for ATP hydrolysis, subunit assembly, or ClpP binding.

\section{Multiple functions of the protein-processing pore}

Some mutations within the conserved GYVG pore motif of ClpX cause general defects in substrate degradation. For example, changing the tyrosine in this motif to alanine caused a total loss of activity for all substrates 
tested in vivo and in vitro. However, because of the global defects caused by the Y153A mutation in ClpX and by similar mutations in HslU and FtsH (Song et al. 2000; Yamada-Inagawa et al. 2003), it has not been possible to establish the mechanism(s) by which these pore mutations affect enzymatic activity.

In contrast, analysis of ClpX mutants with substitutions for the valine of the GYVG pore motif have provided new insights about pore function. This valine was found to be somewhat more tolerant than the tyrosine to mutations. Importantly, experiments with the V154F ClpX mutant reveal that the conserved pore motif participates in two distinct steps of substrate interactions with ClpX and does so in a manner that depends on the identity of the substrate degradation tag. The V154F mutant was specifically and severely defective in degrading substrates carrying $\mathrm{C}$-motif 1 recognition tags. (The $\mathrm{ClpX}^{\mathrm{VF}}$ mutant is also defective in degradation of HsdR on the basis of the in vivo restriction alleviation assay. However, how HsdR is recognized is not well understood; thus, we cannot yet conclude whether this defect is also due to poor recognition of C-motif 1-like peptide motifs or a different defect.) Direct peptide-binding studies, as well as kinetic analysis, established that this defect is caused principally by an approximate 150-fold weakening of ClpX binding to the degradation tag of these substrates. Thus, these data reveal that the pore plays a critical role in recognition of C-motif 1 substrates. The pore motif may bind these recognition tags directly. Alternatively, the V154F pore mutation may alter the C-motif 1-binding site in an allosteric fashion. In either case, our results demonstrate that the pore plays a role in the early steps of C-motif 1 substrate recognition. Because proteins carrying other classes of recognition motifs are relatively unaffected by the V154F mutation, we further conclude that ClpX uses at least two distinct modes for recognizing substrates. The simplest explanation for this observation is that ClpX has two or more sites involved in the recognition of distinct classes of substrates, only one of which is affected by the V154F mutation.

In addition to the large effect of the V154F mutation on ClpX binding to C-motif 1 substrates, $\mathrm{V}_{\max }$ for ClpXP degradation of these substrates was also substantially reduced. As a consequence, even when the binding defect was suppressed by use of high concentrations of ssrAtagged substrate, the rate of protein degradation was still substantially slower than observed with wild-type ClpXP. Slow degradation was also observed with saturating concentrations of a denatured substrate, and thus, cannot be attributed to a reduced rate of enzyme-catalyzed protein unfolding.

What is the nature of the $\mathrm{V}_{\max }$ defect for $\mathrm{ClpX}^{\mathrm{VF}}$ ? Because this mutant degrades some substrates at near wildtype rates (Fig. 3C and legend), a general defect in protein translocation from ClpX to ClpP seems highly unlikely. Moreover, although it makes sense for some parts of the ClpX pore to be involved in translocation, it is difficult to construct models in which general translocation would depend on the sequence of the recognition tag. To explain why the $\mathrm{V}_{\max }$ defect of $\mathrm{ClpX} \mathrm{VF}^{\mathrm{VF}}$ is specific for C-motif 1 substrates, we propose that this mutant is inefficient in engaging these bound substrates for enzymatic processing. Inefficient substrate engagement could also result in a slippery enzyme-substrate complex that easily becomes disengaged during the initial steps of pro- tein unfolding or translocation. An engagement step of this type has also been proposed on the basis of the properties of ClpX mutations that effect communication between subunit-subunit contacts and the ATPase active site (Joshi et al. 2003). Thus, our current view of the mechanism of ClpXP degradation involves five distinct steps as follows: (1) bimolecular binding of ClpX to the substrate recognition tag; (2) unimolecular tag-dependent substrate engagement; $(3)$ the unfolding of native substrates; (4) translocation of the denatured polypeptide into ClpP; and (5) peptide-bond hydrolysis. On the basis of the studies reported here, the GYVG ClpX pore motif appears to play critical roles in the first two steps for C-motif 1 substrates.

\section{Materials and methods}

Molecular biology

Plasmids encoding ClpX $\mathrm{XF}^{\mathrm{VF}}$ and $\mathrm{ClpX} \mathrm{X}^{\mathrm{YA}}$ were constructed by introducing mutations using Quik-Change (Stratagene) into genes for wild-type ClpX and ClpX-His ${ }_{6}$, respectively (Levchenko et al. 1997). The presence of the expected mutations was confirmed by DNA sequencing. Mu plating and restriction alleviation assays were performed as described previously (Kim et al. 2001).

Proteins

$\mathrm{ClpP}, \mathrm{ClpX}, \mathrm{ClpX}{ }^{\mathrm{VF}}, \mathrm{ClpX}-\mathrm{His}_{6}$, and $\mathrm{ClpX}^{\mathrm{YA}}-\mathrm{His}_{6}$ were purified using published protocols (Levchenko et al. 1997; Kim et al. 2000; Flynn et al. 2001). In some $\mathrm{ClpX}^{\mathrm{VF}}$ purifications, the hydroxyapaptite chromatography step was omitted. SspB was a gift from David Wah (Massachusetts Institute of Technology, Cambridge, MA). ${ }^{35}$ S-labeled Arc fusion proteins were purified as reported previously (Burton et al. 2001), with the exception that the storage buffer contained $10 \mathrm{mM}$ Tris $(\mathrm{pH} 7.5), 100 \mathrm{mM}$ $\mathrm{NaCl}, 0.2 \mathrm{mM}$ EDTA. The Arc-ssrA used for the experiment shown in Figure 5 was buffer exchanged into water, lyophilized, and resuspended in $10 \mathrm{mM}$ Tris ( $\mathrm{pH} 7.5), 10 \mathrm{mM} \mathrm{KCl}, 0.2 \mathrm{mM}$ EDTA. Unlabeled $\lambda \mathrm{O}$-Arc, IscS-Arc, DksA-Arc were gifts from Julia Flynn (Massachusetts Institute of Technology, Cambridge, MA). The Arc fusion substrates carried the following tag sequences: Arc-ssrA AANDE NYALAA, Arc$\mathrm{Crl}^{123-133}$ FRDEPVKLTA, Arc-Gcp ${ }^{327-337}$ RWPLAELPAA, Arc-MuA ${ }^{653-663}$ ILEQNRRKKAI, $\lambda \mathrm{O}^{2-12}$-Arc TNTAKILNFGR, IscS ${ }^{1-11}$-Arc MKLPIYLDYSA, and DksA ${ }^{1-11}$-Arc MQEGQNRKTSS. Titin-V13P-I27-ssrA was labeled by growth of cells in the presence of $\left[{ }^{35} \mathrm{~S}\right]$ methionine, purified, and carboxymethylated as described (Kenniston et al. 2003). A peptide (fluoresceinNKKGRHGAANDENYALAA-COOH) containing the ssrA-tag was synthesized by MIT Biopolymers, desalted, and purified on an LC-10AD-VP HPLC column (Shimadzu).

Biochemical assays

Degradation of ${ }^{35} \mathrm{~S}$-labeled Arc fusion proteins, ${ }^{35} \mathrm{~S}$-labeled CM-titinssrA, and unlabeled GFP-ssrA was performed at $30^{\circ} \mathrm{C}$ in degradation buffer [25 mM HEPES-KOH (pH 7.6), $5 \mathrm{mM} \mathrm{KCl,} 5 \mathrm{mM} \mathrm{MgCl}_{2}, 0.032 \%$ NP-40, and 10\% glycerol] with an ATP regenerating system (4 mM ATP, $16 \mathrm{mM}$ creatine phosphate, and $0.32 \mathrm{mg} / \mathrm{mL}$ creatine kinase). Reactions contained $0.3 \mu \mathrm{M} \mathrm{ClpX}$ (or variants), $0.8 \mu \mathrm{M} \mathrm{ClpP}_{14}$, and protein substrates. When present, the SspB concentration was $0.5 \mu \mathrm{M}$. Degradation of ${ }^{35} \mathrm{~S}$-labeled substrates was assayed by changes in TCA-soluble radioactivity as described (Burton et al. 2001). Binding of the ssrA peptide (100 $\mathrm{nM}$ ) to ClpX was assayed in degradation buffer plus $5 \mathrm{mM} \mathrm{ATP} \gamma \mathrm{S}$ at $30^{\circ} \mathrm{C}$ by changes in fluorescence polarization (excitation $467 \mathrm{~nm}$; emission 511 nm) using a Fluoromax-2 instrument (ISA, Jobin-Yvon) and DATAMAX software. ClpX and ATP $\gamma \mathrm{S}$ were preincubated at $30^{\circ} \mathrm{C}$, added to a cuvette prewarmed to $30^{\circ} \mathrm{C}$ prior to the addition of peptide. After polarization stabilized, the signal was averaged for $5 \mathrm{~min}$. ATP hydrolysis was measured using a coupled assay as described (Kim et al. 2001).

\section{Acknowledgments}

We thank Julia Flynn, Elizabeth Courtenay, and Tanya Sokolsky for helpful discussions and David Wah and John Kenniston (Massachusetts Institute of Technology, Cambridge, MA) for gifts of materials. Supported by the Howard Hughes Medical Institute (HHMI) and NIH grant AI-16892.

The publication costs of this article were defrayed in part by payment 
of page charges. This article must therefore be hereby marked "advertisement" in accordance with 18 USC section 1734 solely to indicate this fact.

\section{References}

Burton, B.M. and Baker, T.A. 2003. Mu transpososome architecture ensures that unfolding by ClpX or proteolysis by ClpXP remodels but does not destroy the complex. Chem. Biol. 10: 463-472.

Burton, R.E., Siddiqui, S.M., Kim, Y.I., Baker, T.A., and Sauer, R.T. 2001 Effects of protein stability and structure on substrate processing by the ClpXP unfolding and degradation machine. EMBO J. 20: 30923100 .

Flynn, J.M., Levchenko, I., Seidel, M., Wickner, S.H., Sauer, R.T., and Baker, T.A. 2001. Overlapping recognition determinants within the ssrA degradation tag allow modulation of proteolysis. Proc. Natl. Acad. Sci. 98: 10584-10589.

Flynn, J.M., Neher, S.B., Kim, Y.I., Sauer, R.T., and Baker, T.A. 2003. Proteomic discovery of cellular substrates of the ClpXP protease reveals five classes of ClpX-recognition signals. Mol. Cell 11: 671-683.

Gottesman, S., Roche, E., Zhou, Y., and Sauer, R.T. 1998. The ClpXP and ClpAP proteases degrade proteins with carboxy-terminal peptide tails added by the SsrA-tagging system. Genes \& Dev. 12: 1338-1347.

Hengge, R. and Bukau, B. 2003. Proteolysis in prokaryotes: Protein quality control and regulatory principles. Mol. Microbiol. 49: 1451-1462.

Joshi, S.A., Baker, T.A., and Sauer, R.T. 2003. C-terminal domain mutations in ClpX uncouple substrate binding from an engagement step required for unfolding. Mol. Microbiol. 48: 67-76.

Keiler, K.C., Waller, P.R., and Sauer, R.T. 1996. Role of a peptide tagging system in degradation of proteins synthesized from damaged messenger RNA. Science 271: 990-993.

Kenniston, J.A., Baker, T.A., Fernandez, J.M., and Sauer, R.T. 2003. Linkage between ATP consumption and mechanical unfolding during the protein processing reactions of an AAA+ degradation machine. Cell 114: $511-520$.

Kim, D.Y. and Kim, K.K. 2003. Crystal structure of ClpX molecular chaperone from Helicobacter pylori. J. Biol. Chem. 278: 50664-50670.

Kim, Y.I., Burton, R.E., Burton, B.M., Sauer, R.T., and Baker, T.A. 2000. Dynamics of substrate denaturation and translocation by the ClpXP degradation machine. Mol. Cell 5: 639-648.

Kim, Y.I., Levchenko, I., Fraczkowska, K., Woodruff, R.V., Sauer, R.T., and Baker, T.A. 2001. Molecular determinants of complex formation between Clp/Hsp100 ATPases and the ClpP peptidase. Nat. Struct. Biol. 8: 230-233.

Kruklitis, R., Welty, D.J., and Nakai, H. 1996. ClpX protein of Escherichia coli activates bacteriophage $\mathrm{Mu}$ transposase in the strand transfer complex for initiation of $\mathrm{Mu}$ DNA synthesis. EMBO J. 15: 935-944.

Lee, C., Schwartz, M.P., Prakash, S., Iwakura, M., and Matouschek, A. 2001. ATP-dependent proteases degrade their substrates by processively unraveling them from the degradation signal. Mol. Cell 7: 627637.

Levchenko, I., Luo, L., and Baker, T.A. 1995. Disassembly of the $\mathrm{Mu}$ transposase tetramer by the ClpX chaperone. Genes \& Dev. 9: 23992408.

Levchenko, I., Smith, C.K., Walsh, N.P., Sauer, R.T., and Baker, T.A. 1997. PDZ-like domains mediate binding specificity in the Clp/ Hsp100 family of chaperones and protease regulatory subunits. Cell 91: 939-947.

Levchenko, I., Seidel, M., Sauer, R.T., and Baker, T.A. 2000. A specificityenhancing factor for the ClpXP degradation machine. Science 289: 2354-2356.

Makovets, S., Doronina, V.A., and Murray, N.E. 1999. Regulation of endonuclease activity by proteolysis prevents breakage of unmodified bacterial chromosomes by type I restriction enzymes. Proc. Natl. Acad. Sci. 96: 9757-9762.

Ortega, J., Singh, S.K., Ishikawa, T., Maurizi, M.R., and Steven, A.C. 2000. Visualization of substrate binding and translocation by the ATP-dependent protease, ClpXP. Mol. Cell 6: 1515-1521.

Singh, S.K., Grimaud, R., Hoskins, J.R., Wickner, S., and Maurizi, M.R. 2000. Unfolding and internalization of proteins by the ATP-dependent proteases ClpXP and ClpAP. Proc. Natl. Acad. Sci. 97: 88988903.
Song, H.K., Hartmann, C., Ramachandran, R., Bochtler, M., Behrendt, R., Moroder, L., and Huber, R. 2000. Mutational studies on HslU and its docking mode with HslV. Proc. Natl. Acad. Sci. 97: 14103-14108.

Wah, D.A., Levchenko, I., Baker, T.A., and Sauer, R.T. 2002. Characterization of a specificity factor for an AAA+ ATPase: Assembly of SspB dimers with ssrA-tagged proteins and the ClpX hexamer. Chem. Biol. 9: $1237-1245$.

Wang, J., Hartling, J.A., and Flanagan, J.M. 1997. The structure of ClpP at 2.3Å resolution suggests a model for ATP-dependent proteolysis. Cell 91: $447-456$.

Wang, J., Song, J.J., Franklin, M.C., Kamtekar, S., Im, Y.J., Rho, S.H., Seong, I.S., Lee, C.S., Chung, C.H., and Eom, S.H. 2001. Crystal structures of the HslVU peptidase-ATPase complex reveal an ATP-dependent proteolysis mechanism. Structure 9: 177-184.

Wawrzynow, A., Wojtkowiak, D., Marszalek, J., Banecki, B., Jonsen, M. Graves, B., Georgopoulos, C., and Zylicz, M. 1995. The ClpX heatshock protein of Escherichia coli, the ATP-dependent substrate specificity component of the ClpP-ClpX protease, is a novel molecular chaperone. EMBO J. 14: 1867-1877.

Weber-Ban, E.U., Reid, B.G., Miranker, A.D., and Horwich, A.L. 1999. Global unfolding of a substrate protein by the Hsp100 chaperone ClpA. Nature 401: 90-93.

Yamada-Inagawa, T., Okuno, T., Karata, K., Yamanaka, K., and Ogura, T. 2003. Conserved pore residues in the AAA protease, $\mathrm{FtsH}$, are important for proteolysis and its coupling to ATP hydrolysis. J. Biol. Chem. 278: 50182-50187. 


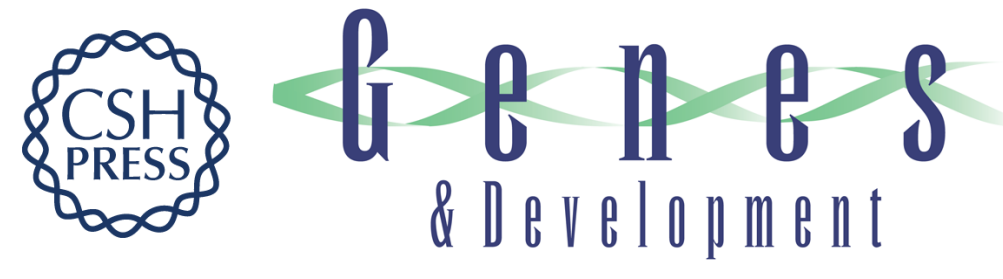

\section{Role of the processing pore of the ClpX AAA+ ATPase in the recognition and engagement of specific protein substrates}

Samia M. Siddiqui, Robert T. Sauer and Tania A. Baker

Genes Dev. 2004, 18:

Access the most recent version at doi:10.1101/gad.1170304

References This article cites 27 articles, 11 of which can be accessed free at: http://genesdev.cshlp.org/content/18/4/369.full.htmI\#ref-list-1

License

Email Alerting

Receive free email alerts when new articles cite this article - sign up in the box at the top Service right corner of the article or click here.

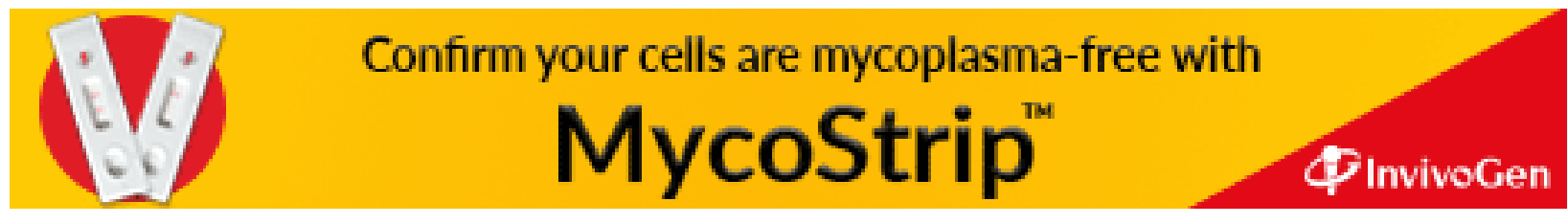

